Specific tagging of the egress-related osmiophilic bodies in the gametocytes of Plasmodium falciparum

Sannella et al. 


\title{
Specific tagging of the egress-related osmiophilic bodies in the gametocytes of Plasmodium falciparum
}

\author{
Anna Rosa Sannella ${ }^{1 \dagger}$, Anna Olivieri $^{{ }^{* \dagger}}$, Lucia Bertuccini ${ }^{2}$, Fabrizio Ferrè ${ }^{3}$, Carlo Severini ${ }^{1}$, Tomasino Pace ${ }^{1}$ and \\ Pietro Alano ${ }^{1}$
}

\begin{abstract}
Background: Gametocytes, the blood stages responsible for Plasmodium falciparum transmission, contain electron dense organelles, traditionally named osmiophilic bodies, that are believed to be involved in gamete egress from the host cell. In order to provide novel tools in the cellular and molecular studies of osmiophilic body biology, a $P$. falciparum transgenic line in which these organelles are specifically marked by a reporter protein was produced and characterized.

Methodology: A P. falciparum transgenic line expressing an 80-residue N-terminal fragment of the osmiophilic body protein Pfg377 fused to the reporter protein DsRed, under the control of pfg377 upstream and downstream regulatory regions, was produced.

Results: The transgenic fusion protein is expressed at the appropriate time and stage of sexual differentiation and is trafficked to osmiophilic bodies as the endogenous Pfg377 protein. These results indicate that a relatively small $\mathrm{N}$-terminal portion of Pfg377 is sufficient to target the DsRed reporter to the gametocyte osmiophilic bodies.

Conclusions: This is the first identification of a P. falciparum aminoacid sequence able to mediate trafficking to such organelles. To fluorescently tag such poorly characterized organelles opens novel avenues in cellular and imaging studies on their biogenesis and on their role in gamete egress.
\end{abstract}

Keywords: Malaria, Plasmodium falciparum, pfg377, Female gametocyte, Osmiophilic body, Subcellular localization, Gamete egress, Trafficking

\section{Background}

Progression of parasite development through diverse intracellular and extracellular forms is accompanied by the biogenesis of several organelles, some of which rhoptries, dense granules and micronemes - are specific of apicomplexan parasites. Functional role of such organelles is being elucidated in key aspects of parasite development such as invasion of and egress from the host cell, and they are increasingly attracting attention as potential targets of anti-parasitic drugs. Functional studies on protein trafficking to such organelles revealed

\footnotetext{
* Correspondence: anna.olivieri@iss.it

+ Contributed equally

'Infectious Diseases Department, Istituto Superiore di Sanità, v.le Regina Elena 299, Rome 00161, Italy

Full list of author information is available at the end of the article
}

that specific aminoacid sequences are necessary for proper organelle targeting [1]. Some of such studies, however, indicated that appropriate timing of expression for some of these proteins can be critical for targeting, as shown in the cases of Plasmodium berghei protein AMA-1 [2] and Plasmodium falciparum proteins RESA and RhopH2 $[3,4]$.

Gametocytes, the blood stages responsible for parasite transmission to the mosquito vector, contain electron dense organelles similar to dense granules, traditionally named osmiophilic bodies [5]. In P. falciparum these oval shaped organelles appear on day 4 of sexual differentiation (stage III) [6] and accumulate in female gametocytes, where they progressively increase in number and reach their subcellular localization under the mature gametocyte surface. Ultrastructural observations showed that 
osmiophilic bodies are connected to the gametocyte surface by ducts and are virtually no longer present after transformation into female gamete, thus supporting the hypothesis of their involvement in gamete egress from the host cell [5,7]. This notion found additional support from functional studies on the P. falciparum protein Pfg377, whose ablation by gene knock out caused defective egress of female gametes [8], and the P. berghei proteins Mdv-1/ Peg3 [9] and PbGEST [10].

In order to provide novel tools in the cellular and molecular studies of the biology of osmiophilic bodies, this work aimed to produce a $P$. falciparum transgenic line, in which a reporter protein fused to a portion of the $p f g 377$ coding sequence was specifically targeted to such organelles.

\section{Methods}

\section{Parasite culture and transfection}

The gametocyte-producing P. falciparum clone 3D7 was used to derive the transgenic line 3D7/pEpi377. Parasites were cultured and synchronized using standard protocols [11]. For transfection, ring stage parasites ( $\sim$ \% parasitaemia) were electroporated with 80 micrograms of purified plasmid DNA as previously described [12] and drug selected with $2.5 \mathrm{nM}$ WR99210 $48 \mathrm{~h}$ later. Resistant parasites appeared after approximately three weeks of selection. Gametocyte production was induced by growing parasites to high parasitaemia, while asexual stages were killed by treatment with $0.05 \mathrm{M} \mathrm{N}$ acetylglucosamine for $72 \mathrm{~h}$ [13]. Gametocyte activation was induced by exposing mature gametocytes to room temperature in incomplete medium at $\mathrm{pH}$ 8.2.

\section{5' RACE analysis}

5' RACE (Rapid Amplification of cDNA Ends) experiments were performed using the Boehringer Mannheim 5'/3' RACE kit, according to the manufacturer's instructions. Briefly, a first strand cDNA was generated with the $p f g 377$ antisense primer $\mathrm{pR}$ (see Additional file 1: Table S1 for all primers used), whose sequence is located $30 \mathrm{bp}$ downstream of the gene ATG codon, using 5 micrograms of total mRNA from 3D7 Percoll purified stage III-IV gametocytes. cDNA, purified in GlassMax columns and $\mathrm{dCTp} / \mathrm{TdT}$ tailed, was used to produce double-stranded DNA by PCR, using the 3' tail specific primer provided by the kit. 5 clones of the resulting PCR products were sequenced.

\section{Plasmid construction}

A $1883 \mathrm{bp}$ fragment, including the putative promoter region and $240 \mathrm{bp}$ of $p f g 377$ coding region, was amplified from 3D7 genomic DNA with primers $\mathrm{p} 1$ and $\mathrm{p} 2$ and cloned into the pHHMC*/3R0.5 plasmid [14], using SacII and XhoI restriction sites. DsRed gene was PCR amplified from plasmid pBac(3xP3RED)AgApy [15], with primers p3 and p4 and cloned in the same plasmid with XhoI and KpnI. A second fragment, 385 bp long, spanning the $p f g 377$ stop codon was amplified from 3D7 genomic DNA with primers p5 and p6 and cloned downstream the DsRed gene using KpnI and NheI restriction sites. Coding sequences were double checked by sequencing, using primers $\mathrm{p} 7$ to $\mathrm{p} 9$. The plasmid was named pEpi377 and the map is shown in Additional file 2: Figure S1.

\section{Immunofluorescence analysis}

Gametocytes were fixed in $4 \%$ paraformaldehyde and blocked in $3 \%$ bovine serum albumin overnight, and were simultaneously reacted with the above anti-Pfg377 $B$ portion serum and a polyclonal rabbit anti-DsRed antiserum (MBL International) respectively diluted 1:500 and 1:100. Smears were then incubated with FITC and TRITC-conjugated anti-rat and anti-rabbit IgG, diluted 1:200, and $2 \mathrm{mg} / \mathrm{ml}$ DAPI for $1 \mathrm{~h}$. Smears were washed in PBS, mounted and examined on a Leitz DMR fluorescent microscope using an oil immersion objective.

\section{Immuno electron microscopy}

Stage IV and V gametocytes from the 3D7/pEpi377 transgenic line were Percoll purified and processed for immuno-electron microscopy according to published protocols [16]. Briefly, samples were fixed overnight at $4^{\circ}$ C with $4 \%$ paraformaldehyde/ $0.1 \%$ glutaraldehyde in 0.1 $M$ sodium cacodylate buffer. Next, the suspension was gently washed in sodium cacodylate buffer, dehydrated in ethanol serial dilutions and embedded in LR White, medium-grade acrylic resin (London Resin Company, UK). The samples were polymerized in a $50^{\circ} \mathrm{C}$ oven for $48 \mathrm{~h}$ and ultrathin sections, collected on gold grids, were sunk in $100 \%$ ethanol for $3 \mathrm{~min}$, immersed in Tris buffer 0.05 $\mathrm{M}$ ( $\mathrm{pH}$ 10.0) in PCR tubes, and then kept at $99^{\circ} \mathrm{C}$ for 30 min using a constant temperature box [17].

For immunostaining, the grids were floated on drops of PBS containing $0.1 \mathrm{M}$ glycine for $10 \mathrm{~min}$, washed with PBS, blocked with $5 \%$ normal goat serum/1\% BSA in PBS for $30 \mathrm{~min}$. For single immunogold-labelling of Ds-Red/Pfg377 fusion protein, ultrathin sections were incubated overnight at $4^{\circ} \mathrm{C}$ with a 1:50 dilution of rabbit polyclonal anti-DsRed serum (MBL International) in PBS/0.1\% BSA/0,05\% TWEEN20 buffer. Samples were then rinsed and incubated for $1 \mathrm{~h}$ with $10 \mathrm{~nm}$ gold-conjugated goat anti-rabbit IgG (SIGMA) (1:50), rinsed again in buffer followed by distilled water and finally air dried. For double immunogold labelling, ultrathin sections were incubated overnight at $4^{\circ} \mathrm{C}$ with a $1: 100$ dilution of mouse polyclonal anti-377-B serum [18] in PBS/ $0.1 \%$ BSA/0,05\% TWEEN20 buffer. After washing, the grids were incubated for $1 \mathrm{~h}$ with $5 \mathrm{~nm}$ gold-conjugated 
goat anti-mouse IgG (Sigma Aldrich) diluted 1:50, rinsed in buffer and further incubated with the above rabbit polyclonal anti-DsRed serum $(1: 100)$ overnight at $4^{\circ} \mathrm{C}$. Ultrathin sections were then rinsed and incubated for 1 $\mathrm{h}$ with $10 \mathrm{~nm}$ gold-conjugated goat anti-rabbit IgG (SIGMA) (1:50), rinsed again in buffer followed by distilled water and air dried. Finally, the samples were stained successively with uranyl acetate $2 \%$ in $\mathrm{H}_{2} \mathrm{O}$ and Reynolds lead citrate solution, and observed with an EM208 Philips transmission electron microscope. As controls, the sections were stained without any heating, without the primary antibody and with the diluted gold anti-mouse IgG, or with the diluted mouse pre-immune serum in place of the first antibody.

\section{Results and discussion}

\section{Regulatory and coding sequences of the pfg377 gene for} reporter construct

In order to produce a plasmid construct expressing a Pfg377 fragment fused to a reporter protein, for possible targeting to female gametocyte osmiophilic bodies, the following regulatory sequences were used. A $1.6 \mathrm{~kb}$ fragment (1643 bp) of genomic region upstream the $p f g 377$ ATG codon and a $0.36 \mathrm{~kb}$ fragment (361 bp) of downstream region from the gene TAA stop codon were amplified and cloned to drive expression of the $p f g 377$ fusion protein. A 5' RACE experiment on total RNA from Percoll purified stage IV gametocytes was preliminarily conducted to investigate length of the 5'UTR. Results showed that all cDNA clones obtained in this experiments extended to nucleotide -294 from the gene start codon, thus indicating that $1.6 \mathrm{~kb}$ of upstream sequences was adequate to contain the $p f g 377$ promoter.

As the first 85 amino acids of the Toxoplasma gondii rhoptry protein ROP1 and the initial 24 of the P. falciparum rhoptry protein $\mathrm{RhopH} 2$ were sufficient to drive fluorescent reporters to the respective organelles $[4,19]$, the sequence encoding the first 80 amino acids from pfg377 start codon was used in the design of the fusion protein. Next to such 80 aminoacids, a repetitive region encoding a Pro-Glu dipeptide repeat was not included in the chimera. In summary, the above upstream and downstream regulatory regions were cloned flanking a sequence encoding the first 80 aminoacids of Pfg377 fused to the DsRed coding sequence (Figure 1). These sequences were cloned in a plasmid containing the $h$-dhfr selection cassette conferring parasite resistance to WR99210, yielding plasmid pEpi377 (Additional file 2: Figure S1).

\section{Stage-specificity, timing and localization of the transgenic Pfg377-DsRed fusion protein}

The pEpi377 plasmid was transfected in the gametocyte producer clone 3D7 and WR99210 resistant transgenic parasites were selected and analysed. Parasites of the

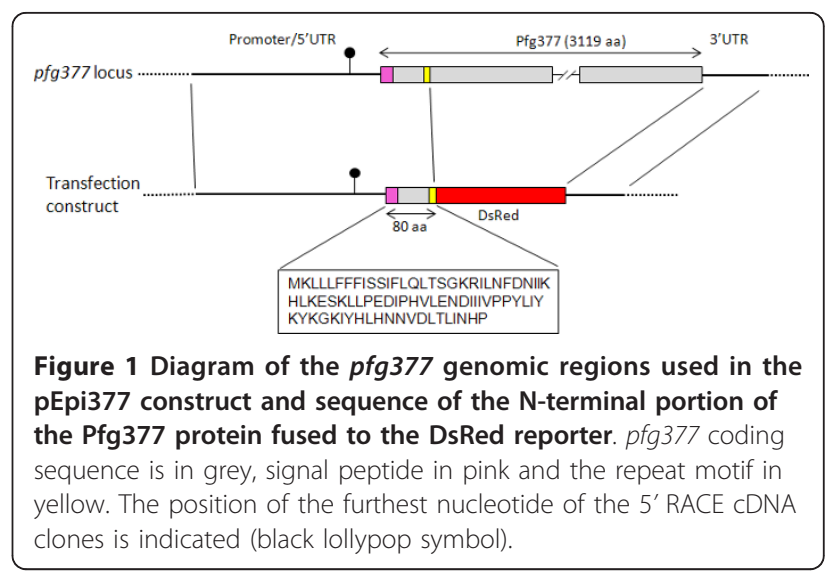

resulting line 3D7/pEpi377 were never seen to fluoresce at any stage of asexual development. A time course of gametocytogenesis in this line was then analysed. Examination of synchronous gametocytes revealed that the round shaped stage I and the crescent shaped stage II gametocytes did not show any fluorescence. A clearly distinguishable fluorescent signal was instead detectable in stage III gametocytes (approximately at day 4 of gametocytogenesis) (Figure 2A), becoming more intense in stage IV and stage $\mathrm{V}$ gametocytes (Figure 2B and 2C). Intriguingly, the fraction of gametocytes showing the fluorescent signal was usually around $10 \%$, noticeably lower than an expected $40 \%$. This estimate was based on the fact that several studies using episomal constructs in gametocytes reported that only $50-60 \%$ sexual parasites express the episomeencoded fluorescent reporter [20], probably because episomes do not segregate to each daughter cell and gametocytes are not affected by the antifolate WR99210. In addition, the fluorescent signal was predicted to appear only in the fraction of female gametocytes, routinely observed to be $80 \%$ in 3D7. A reason for the lower fraction of fluorescent gametocytes observed in the 3D7/pEpi377 line might be that a higher plasmid copy number is required for detection of the Pfg377/DsRed fluorescent signal compared to other fusion proteins. Increasing the concentration of WR99210 to possibly amplify the plasmid copy number however did not result in a higher percent of DsRed fluorescent gametocytes.

Analysis of the pattern of fluorescence in the Pfg377/ DsRed-positive gametocytes clearly showed that the fluorescent signal was concentrated in dots evenly distributed in the gametocyte cytoplasm (Figure 2). This feature was reminiscent of the appearance of Pfg377positive granules in immunofluorescence of female gametocytes, and thus consistent with the possibility that the fluorescence localized to osmiophilic bodies. Immunofluorescence analysis of stage IV gametocytes with antibodies specific for DsRed and for the B portion of Pfg377, which is not included in the Pfg377/DsRed 


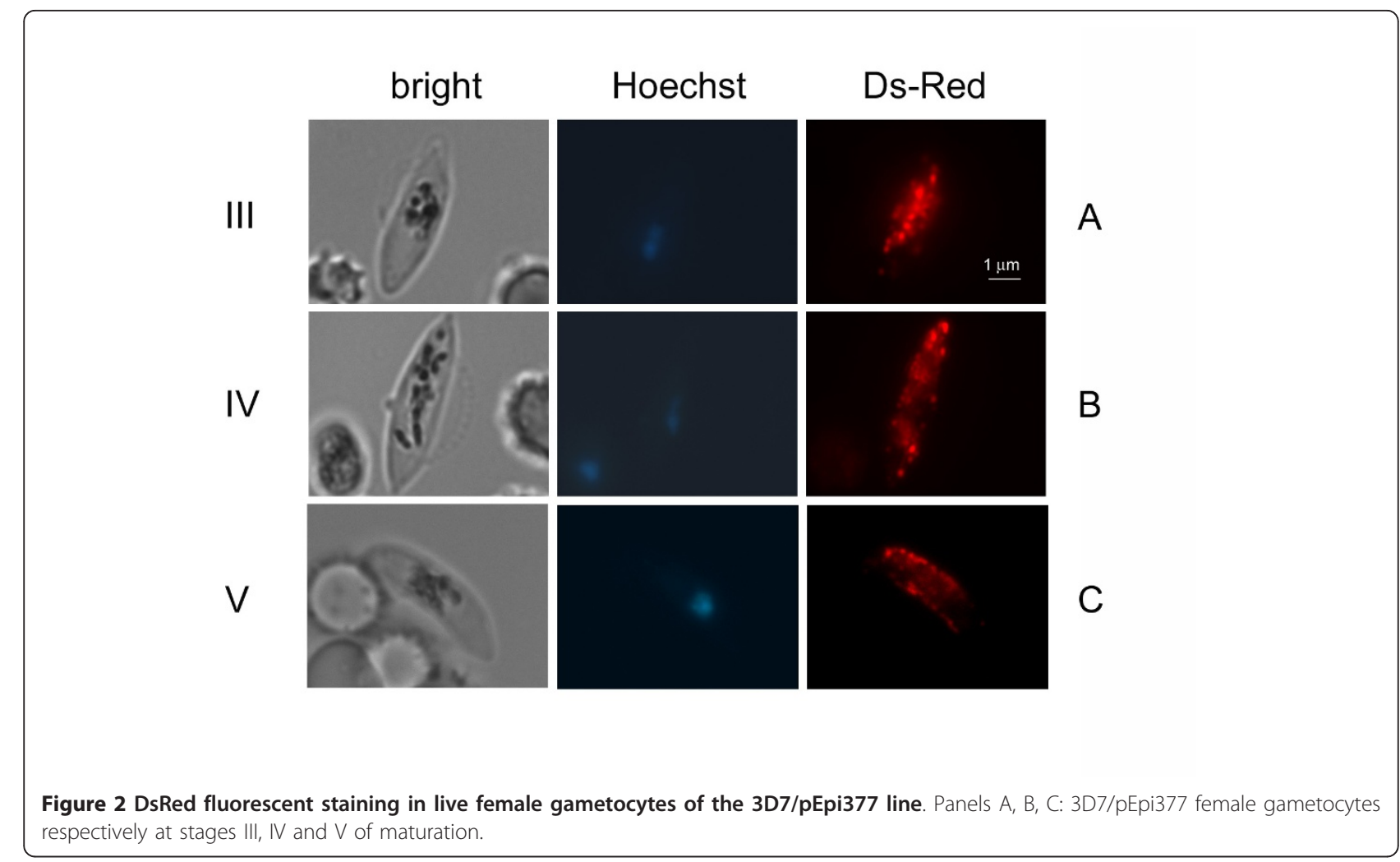

chimera [18], showed that the DsRed-specific signal largely co-localized with that of the anti-Pfg377 antiserum (Figure 3). Partial co-localization is due to the different intensity of the fluorescent signals, being the anti-DsRed signal in general lower, probably due to the lower amount of protein. Finally, as Pfg377 antibodies specifically label female gametocytes [21], the observation that anti-DsRed reactivity was restricted to anti-Pfg377 positive parasites confirmed that the reporter protein is expressed only in female gametocytes.

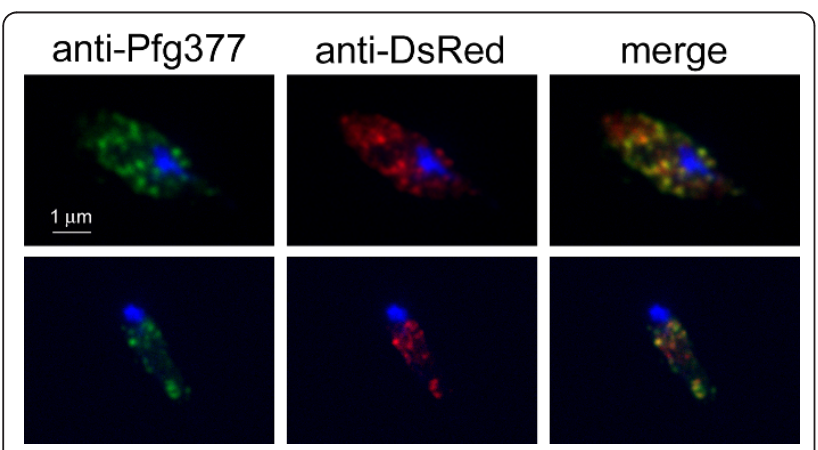

Figure 3 IFA of 3D7/pEpi377 parasites. The transgenic Pfg377/ DsRed fusion protein, detected by anti-DsRed (red) colocalizes with the endogenous Pfg377 (green) in female gametocytes. Nuclei are stained with DAPI.
Ultrastructural analysis of Pfg377-DsRed localization

In order to unambiguously confirm Pfg377/DsRed colocalization, stage IV and V gametocytes from the 3D7/ pEpi377 line were Percoll purified and fixed sections were prepared for immuno-electron microscopy analysis. In one experiment, these were reacted with antibodies against DsRed alone (Figure 4A) and in another one they were simultaneously reacted with antibodies specific for the Pfg377 B portion and for the DsRed moiety of the fusion protein, which were revealed with two secondary antibody-conjugated gold particles of respectively 5 and $10 \mathrm{~nm}$ (Figure 4B). Representative images of the reacted gametocyte sections clearly show that the antiDsRed rabbit antibodies specifically stain osmiophilic bodies (Figure 4A) and analysis of the double immunoelectron microscopy showed that distribution of the different size gold particles almost completely co-localized (Figure 4B). These results in conclusion indicate that the Pfg377/DsRed fusion, after being produced at the appropriate time and stage of sexual differentiation, is trafficked to female gametocyte osmiophilic bodies as the endogenous Pfg377 protein.

\section{Analysis of the transgenic Pfg377/DsRed fusion protein during gamete emergence}

As osmiophilic bodies are reported to disappear in female gametes at egress, the expression pattern of the 


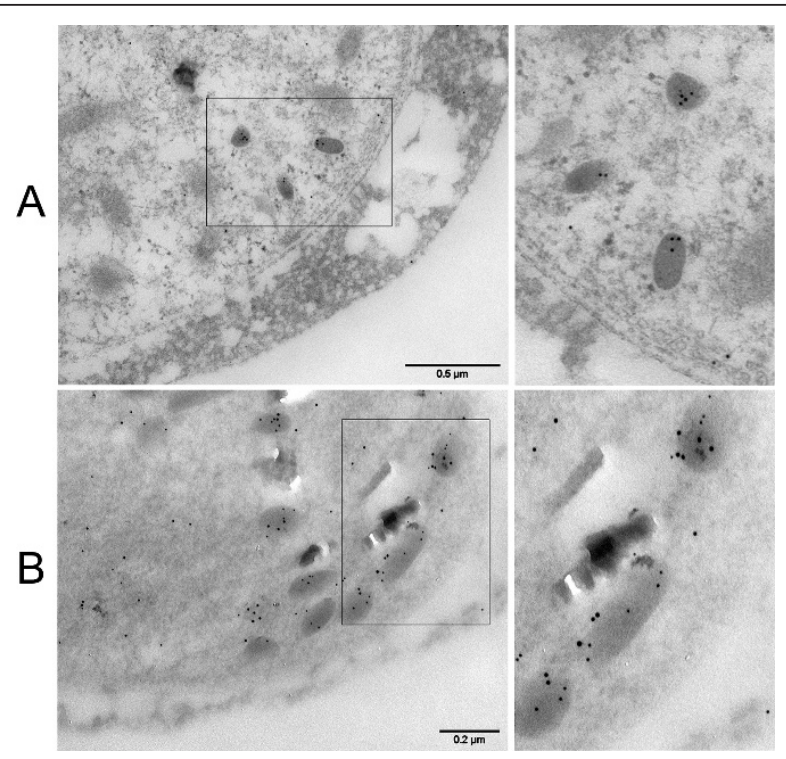

Figure 4 Immunoelectron microscopy analysis of Pfg377/DsRed fusion protein localization in stage IV female gametocytes. Panel A: 5 $\mathrm{nm}$ gold particles specific for DsRed specifically localize on electron dense osmiophilic bodies. Panel B: $5 \mathrm{~nm}$ gold particles specific for the antiPfg377 serum and $10 \mathrm{~nm}$ gold particles specific for the anti-DsRed antiserum, co-localize on osmiophilic bodies.

transgenic Pfg377/DsRed fusion protein was analysed during this process. 3D7/epi377 stage $\mathrm{V}$ gametocytes were triggered to transform into gametes and the fluorescence pattern was analysed in live parasites collected before and at 3, 10 and 20 minutes post induction. As soon as rounding up was observed the fluorescent dots appeared to be more loosely associated to the female gamete membrane, starting to quiver and gradually disappearing. $10 \mathrm{~min}$ after induction, fluorescence was still detectable, although it was weaker than in the elongated gametocyte and its pattern was not granular but diffuse around the cell, progressively fading with time (Figure 5). In conclusion, mobilization and progressive disappearance of Pfg377/DsRed fluorescent dots and their redistribution from an intracellular to an extracellular localization in the transition from stage $\mathrm{V}$ female gametocytes to gametes indicates that the fluorescence signal follows the same fate of osmiophilic bodies in female gametogenesis and is consistent with the disappearance of Pfg377 signal in immunofluorescence assays (see Additional file 3: Figure S2). As this process has been so far described only by electron microscopy, this result

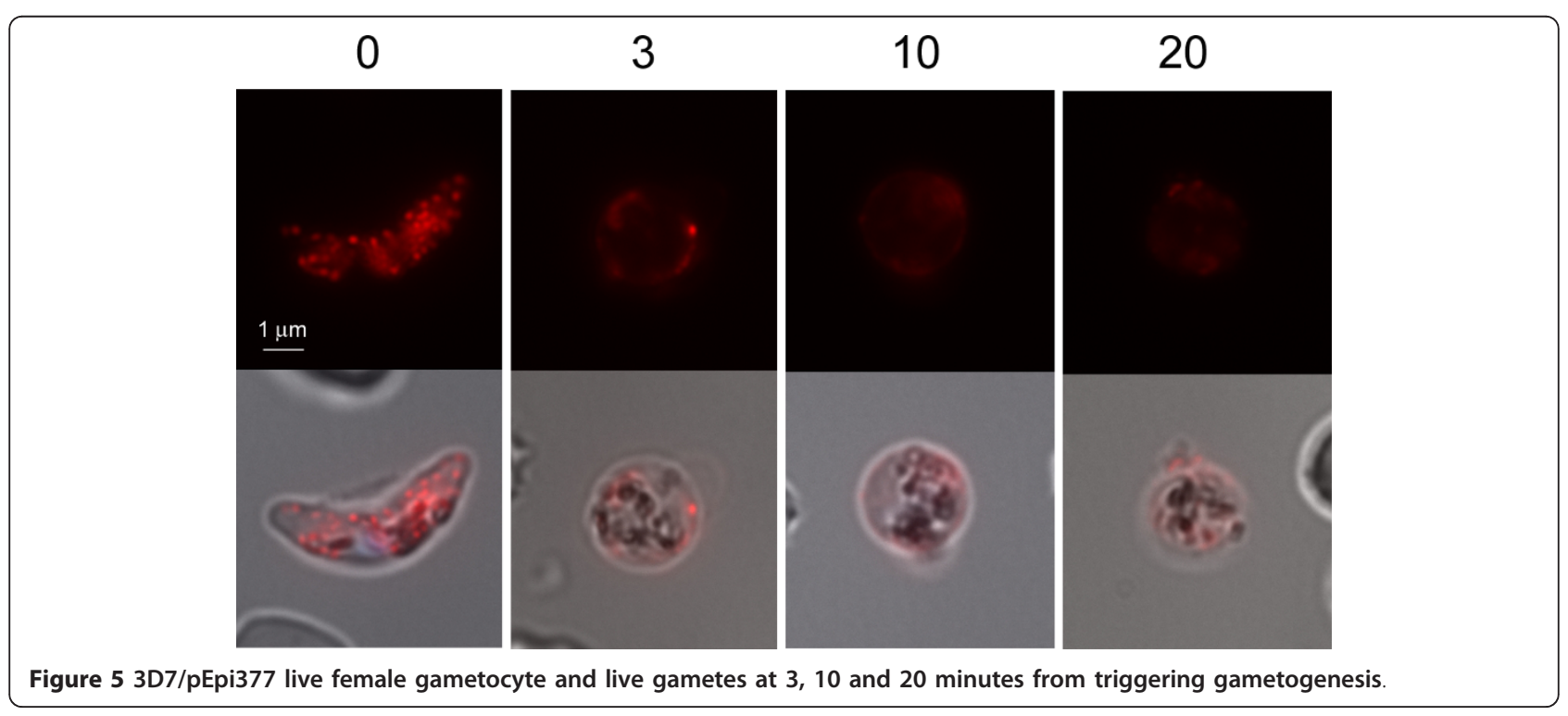


represents the first dynamic analysis of these events in P. falciparum female gametogenesis.

\section{The N-terminal portion of Pfg377 is sufficient for targeting a fluorescent reporter to female gametocyte osmiophilic bodies}

The experiments reported here indicate that the first 80 aminoacids of Pfg377 are sufficient to target the DsRed reporter to female gametocyte osmiophilic bodies. This is to our knowledge the first identification of a P. falciparum aminoacid sequence able to mediate trafficking to such organelles, which are comparatively less characterized than similar electron dense organelles such as rhoptries, micronemes and dense granules in other parasite developmental stages. Molecular composition of osmiophilic bodies is also much less characterized than that of the above organelles, as only the gametocyte proteins Pfg377 and PfMdv-1/Peg3, the latter however abundantly present also in additional membrane compartments [22,23], have been so far localized in such organelles in P. falciparum. Virtually nothing is known on biogenesis of and protein trafficking to osmiophilic bodies. In general, trafficking to organelles of the apical complex such as rhoptries, dense granules and micronemes have been mainly investigated in asexual stages of Plasmodium and T. gondii, and requirement of specific sequences in proper organelle localization is still poorly understood. From studies on vesicular trafficking in lysosome biogenesis in higher eukaryotes it results that $\mathrm{C}$-terminal portions of lysosomal proteins are recognized in the Golgi by cytoplasmic adaptor proteins that mediate their trafficking to such organelles. In $T$. gondii, the microneme proteins MIC2 and MIC6 and the rhoptry protein ROP2 reach their organelle destination with such a mechanism [24-26]. At odds with the picture emerging from the above studies, functional analyses of fusion protein localization indicated instead that in $T$. gondii the first 85 aminoacids of the rhoptry protein ROP1 are sufficient to ensure proper organelle targeting [19], and in P. falciparum the initial 24 residues of the rhoptry protein $\mathrm{RhopH} 2$ are able to drive localization of a fluorescent reporter to such organelles [4]. The work presented here further supports the hypothesis that a signal peptide and a relatively limited $\mathrm{N}$-terminal portion may be sufficient to specifically traffic a parasite protein to the gametocyte osmiophilic bodies.

As only three proteins have been so far positively localized in osmiophilic bodies (Pfg377, Pf Mdv-1/Peg3, PfGEST), a comparative sequence analysis to identify functionally conserved motifs is difficult. Nevertheless, both this approach and structural modeling analysis were undertaken to predict motifs functionally involved in trafficking to such organelles (see Additional file 4).
However, the motifs predicted by computational analysis lack in specificity, thus suggesting that a higher number of osmiophilic body-associated proteins is needed to identify the determinants of osmiophilic body localization.

The positive identification presented here of a parasite sequence targeting proteins to female gametocyte osmiophilic bodies is a relevant improvement in the ongoing studies on protein trafficking in parasite sexual differentiation. In addition, the possibility to fluorescently tag such poorly characterized organelles opens novel avenues in cellular and imaging studies on their biogenesis and on their role in gamete egress.

\section{Additional material}

Additional file 1: Table S1. Primers used for plasmid construction and sequencing.

Additional file 2: Figure S1. Map of the pEpi377 plasmid.

Additional file 3: Figure S2. Immunofluorescence assay with antiPfg377 antibodies on a gametocyte culture $10 \mathrm{~min}$ after induction of gametogenesis. Parasites were fixed in $4 \%$ paraformaldehyde and permeabilized with $1 \%$ Triton X-100. Nuclei were stained with DAPI. Images $A$ and $B$ were collected on the same smear with the same exposure time. A. Residual mature gametocyte. B. Female gamete.

Additional file 4: Identification of sequence motifs shared by osmiophilic body-associated proteins [27-33].

\section{Acknowledgements}

Prof. G. Girelli, University of Rome "La Sapienza", is gratefully acknowledged for the gift of human erythrocytes. This work was funded by EU FP7 Projects MALSIG (Contract 223044) and NoE EVIMalaR, and the Italy-USA Collaborative Project from Italian Ministry of Health "Membrane dynamics of Plasmodium sexual stages as potential target of malaria transmission blocking intervention".

\section{Author details}

${ }^{1}$ Infectious Diseases Department, Istituto Superiore di Sanità, v.le Regina Elena 299, Rome 00161, Italy. ${ }^{2}$ Health Technologies Department, University of Rome "Tor Vergata", Via della Ricerca Scientifica S.n.c, Rome 00133, Italy. ${ }^{3}$ Centre for Molecular Bioinformatics (CBM), University of Rome "Tor Vergata", Via della Ricerca Scientifica s.n.c, Rome 00133, Italy.

\section{Authors' contributions}

ARS carried out the molecular and cell biology experiments and participated in the study design. AO and PA conceived the study, participated in its coordination and drafted the manuscript. LB carried out the electron microscopy experiments. FF carried out the bioinformatic analysis. CS carried out the $5^{\prime}$ race experiments. TP participated in the design of the study and helped to generate the plasmid. All authors read and approved the final manuscript.

\section{Competing interests}

The authors declare that they have no competing interests.

Received: 22 December 2011 Accepted: 27 March 2012

Published: 27 March 2012

\section{References}

1. Kats LM, Cooke BM, Coppel RL, Black CG: Protein trafficking to apical organelles of malaria parasites - building an invasion machine. Traffic 2008, 9:176-186. 
2. Kocken $\mathrm{CH}$, van der Wel $\mathrm{AM}$, Dubbeld MA, Narum $\mathrm{DL}$, van de Rijke FM, van Gemert GJ, van der Linde X, Bannister LH, Janse C, Waters AP, Thomas AW: Precise timing of expression of a Plasmodium falciparum-derived transgene in Plasmodium berghei is a critical determinant of subsequent subcellular localization. J Biol Chem 1998, 273:15119-15124.

3. Rug M, Wickham ME, Foley M, Cowman AF, Tilley L: Correct promoter control is needed for trafficking of the ring-infected erythrocyte surface antigen to the host cytosol in transfected malaria parasites. Infect Immun 2004, 72:6095-6105.

4. Ghoneim A, Kaneko O, Tsuboi T, Torii M: The Plasmodium falciparum RhopH2 promoter and first 24 amino acids are sufficient to target proteins to the rhoptries. Parasitol Int 2007, 56:31-43.

5. Bannister LH, Margos G, Hopkins JM: Making a home for Plasmodium post-genomics: ultrastructural organisation of the blood stages. In Molecular Approaches to Malaria. Edited by: Sherman I. Washington DC USA: ASM Press; 2005:.

6. Hawking F, Wilson ME, Gammage K: Evidence for cyclic development and short-lived maturity in the gametocytes of Plasmodium falciparum. Trans R Soc Trop Med Hyg 1971, 65:549-559.

7. Alano P: Molecular approaches to monitor parasite genetic complexity in the transmission of Plasmodium falciparum malaria. Parassitologia 2005, 47:199-203.

8. de Koning-Ward TF, Olivieri A, Bertuccini L, Hood A, Silvestrini F, Charvalias K, Berzosa Diaz P, Camarda G, McElwain TF, Papenfuss T, Healer J, Baldassarri L, Crabb BS, Alano P, Ranford-Cartwright LC: The role of osmiophilic bodies and Pfg377 expression in female gametocyte emergence and mosquito infectivity in the human malaria parasite Plasmodium falciparum. Mol Microbiol 2008, 67:278-290.

9. Ponzi M, Siden-Kiamos I, Bertuccini L, Curra C, Kroeze H, Camarda G, Pace T, Franke-Fayard B, Laurentino EC, Louis C, Waters AP, Janse CJ, Alano P: Egress of Plasmodium berghei gametes from their host erythrocyte is mediated by the MDV-1/PEG3 protein. Cell Microbiol 2009, 11:1272-1288.

10. Talman AM, Lacroix C, Marques SR, Blagborough AM, Carzaniga R, Menard R, Sinden RE: PbGEST mediates malaria transmission to both mosquito and vertebrate host. Mol Microbiol 2011, 82:462-474.

11. Trager W, Jensen JB: Human malaria parasites in continuous culture. Science 1976, 193:673-675.

12. Fidock DA, Wellems TE: Transformation with human dihydrofolate reductase renders malaria parasites insensitive to WR99210 but does not affect the intrinsic activity of proguanil. Proc Natl Acad Sci USA 1997 94:10931-10936.

13. Gupta SK, Schulman S, Vanderberg JP: Stage-dependent toxicity of Nacetyl-glucosamine to Plasmodium falciparum. J Protozool 1985, 32:91-95.

14. Pace T, Olivieri A, Sanchez M, Albanesi V, Picci L, Siden Kiamos I, Janse CJ, Waters AP, Pizzi E, Ponzi M: Set regulation in asexual and sexual Plasmodium parasites reveals a novel mechanism of stage-specific expression. Mol Microbiol 2006, 60:870-882.

15. Lombardo F, Lycett GJ, Lanfrancotti A, Coluzzi M, Arca B: Analysis of apyrase $5^{\prime}$ upstream region validates improved Anopheles gambiae transformation technique. BMC Res Notes 2009, 2:24.

16. Newman GR: LR white embedding medium for colloidal gold methods. In Colloidal gold - principles, methods and applications. Volume 2. Edited by: Hayat MA. New York: Academic Press; 1989.

17. Saito N, Konishi K, Takeda H, Kato M, Sugiyama T, Asaka M: Antigen retrieval trial for post-embedding immunoelectron microscopy by heating with several unmasking solutions. J Histochem Cytochem 2003, 51:989-994.

18. Alano P, Read D, Bruce M, Aikawa M, Kaido T, Tegoshi T, Bhatti S, Smith DK, Luo C, Hansra S, Carter R, Elliott JF: COS cell expression cloning of Pfg377, a Plasmodium falciparum gametocyte antigen associated with osmiophilic bodies. Mol Biochem Parasitol 1995, 74:143-156.

19. Striepen B, Soldati D, Garcia-Reguet N, Dubremetz JF, Roos DS: Targeting of soluble proteins to the rhoptries and micronemes in Toxoplasma gondii. Mol Biochem Parasitol 2001, 113:45-53.

20. Olivieri A, Silvestrini F, Sanchez M, Alano P: A 140-bp AT-rich sequence mediates positive and negative transcriptional control of a Plasmodium falciparum developmentally regulated promoter. Int J Parasitol 2008, 38:299-312.

21. Severini C, Silvestrini F, Sannella A, Barca S, Gradoni L, Alano P: The production of the osmiophilic body protein Pfg377 is associated with stage of maturation and sex in Plasmodium falciparum gametocytes. Mol Biochem Parasitol 1999, 100:247-252.

22. Lanfrancotti $A$, Bertuccini $L$, Silvestrini $F$, Alano P: Plasmodium falciparum: mRNA co-expression and protein co-localisation of two gene products upregulated in early gametocytes. Exp Parasitol 2007, 116:497-503.

23. Furuya T, Mu J, Hayton K, Liu A, Duan J, Nkrumah L, Joy DA, Fidock DA, Fujioka H, Vaidya AB, Wellems TE, Su XZ: Disruption of a Plasmodium falciparum gene linked to male sexual development causes early arrest in gametocytogenesis. Proc Natl Acad Sci USA 2005, 102:16813-16818.

24. Di Cristina M, Spaccapelo R, Soldati D, Bistoni F, Crisanti A: Two conserved amino acid motifs mediate protein targeting to the micronemes of the apicomplexan parasite Toxoplasma gondii. Mol Cell Biol 2000, 20:7332-7341.

25. Reiss M, Viebig N, Brecht S, Fourmaux MN, Soete M, Di Cristina M, Dubremetz JF, Soldati D: Identification and characterization of an escorter for two secretory adhesins in Toxoplasma gondii. J Cell Biol 2001, 152:563-578.

26. Hoppe HC, Ngo HM, Yang M, Joiner KA: Targeting to rhoptry organelles of Toxoplasma gondii involves evolutionarily conserved mechanisms. Nat Cell Biol 2000, 2:449-456.

27. Bryson K, McGuffin LJ, Marsden RL, Ward JJ, Sodhi JS, Jones DT: Protein structure prediction servers at University College London. Nucleic Acids Res 2005, , 33 Web Server: W36-W38.

28. Bailey $\mathrm{TL}$, Elkan C: Fitting a mixture model by expectation maximization to discover motifs in biopolymers. Proc Int Conf Intell Syst Mol Biol 1994, 2:28-36.

29. Grant $C E$, Bailey $T L$, Noble WS: FIMO: scanning for occurrences of a given motif. Bioinformatics 2011, 27(7):1017-1018.

30. Pizzi $E$, Frontali $C$ : Low-complexity regions in Plasmodium falciparum proteins. Genome Res 2001, 11(2):218-229.

31. Camacho C, Coulouris G, Avagyan V, Ma N, Papadopoulos J, Bealer K, Madden TL: BLAST+: architecture and applications. BMC Bioinformatics 2009, 10:421.

32. Petersen TN, Brunak S, von Heijne G, Nielsen H: SignalP 4.0: discriminating signal peptides from transmembrane regions. Nat Methods 2011, 8(10):785-786

33. Gatei W, Kariuki S, Hawley W, ter Kuile F, Terlouw D, Phillips-Howard P, Nahlen B, Gimnig J, Lindblade K, Walker E, et al: Effects of transmission reduction by insecticide-treated bed nets (ITNs) on parasite genetics population structure: I. The genetic diversity of Plasmodium falciparum parasites by microsatellite markers in western Kenya. Malar J 2010, 9:353.

\section{doi:10.1186/1475-2875-11-88}

Cite this article as: Sannella et al:: Specific tagging of the egress-related osmiophilic bodies in the gametocytes of Plasmodium falciparum. Malaria Journal 2012 11:88.

\section{Submit your next manuscript to BioMed Central and take full advantage of:}

- Convenient online submission

- Thorough peer review

- No space constraints or color figure charges

- Immediate publication on acceptance

- Inclusion in PubMed, CAS, Scopus and Google Scholar

- Research which is freely available for redistribution 